\title{
Estado nutricional de crianças e adolescentes em início de tratamento oncológico em um hospital de referência do nordeste do Brasil
}

\author{
Danielle Erilane Silva Pereira ${ }^{a *}$, Elda Silva Augusto de Andrade ${ }^{\mathrm{a}}$, Francisco Pedrosa ${ }^{\mathrm{b}}$, \\ Janine Maciel Barbosa ${ }^{\mathrm{c}}$
}

anstituto de Medicina Integral Professor Fernando Figueira (IMIP ), ${ }^{\mathrm{b}}$ Associação Médica Brasileira, ${ }^{\mathrm{c}}$ Hospital Universitário Lauro Wanderley

\section{Histórico do Artigo}

Recebido em: 18/03/2017

Aceito em: 29/05/2017

\section{Palavras-chave}

Estado nutricional

Avaliação nutricional

Câncer infantil.

\section{RESUMO}

O presente trabalho teve como objetivo avaliar o estado nutricional de crianças e adolescentes em início de tratamento oncológico. É um estudo do tipo série de casos, realizado com crianças e adolescentes em início de tratamento, internados na Unidade de Oncologia Pediátrica do Instituto de Medicina Integral Prof. Fernando Figueira (IMIP, Brasil, Recife-PE), entre os meses de fevereiro a maio de 2012. A coleta de dados foi realizada até 72 horas após a admissão. Foram coletados dados antropométricos, de composição corporal e bioquímicos. Foram admitidos no período do estudo 30 pacientes. A maioria era do sexo masculino (60\%) com média de idade de 7,1 $\pm 4,3$ anos $\mathrm{O}$ estado nutricional foi avaliado utilizando os parâmetros de peso, altura e idade. Os resultados apontam que houve $4,2 \%, 3,3 \% 16,7 \%$ de déficit nutricional de acordo com os indicadores de P/I (peso/idade), A/I (altura/idade) e IMC/I (índice de massa corporal/ idade) respectivamente. Os dados referentes à composição corporal evidenciam maiores frequência de desnutrição quando comparados à avaliação segundo os escores $\mathrm{Z}$ dos indicadores antropométricos. Encontrou-se, portanto, frequências de desnutrição de $44,8 \%, 57,7 \%$ e $25,9 \%$ para os parâmetros de circunferência do braço (CB), prega cutânea tricipital $(\mathrm{PCT})$ e circunferência muscular do braço $(\mathrm{CMB})$ respectivamente. Com relação à albumina sérica, $48,2 \%$ foram classificados com desnutrição, destes a maioria eram pacientes com tumores hematológicos $(65,5 \%)$. Com a utilização da composição corporal, foi observado maior número de pacientes desnutridos quando comparados aos dados antropométricos. Demonstrando a importância da associação de vários métodos de avaliação para um melhor diagnóstico nutricional

Nutritional status of children and adolescents at the beginning of oncological treatment in a reference hospital in northeast of Brazil

Keywords

Nutritional status

Nutritional assessment

Childhood cancer

*Autor correspondente: dani_erilane@hotmail.com (D. E. S. Pereira)
ABSTRACT - The aim of this work was to assess the nutritional status of children and adolescents in early cancer treatment. This is a Study of case series, carried out with children and adolescents starting treatment hospitalized in the Institute of Medicine Professor Fernando Figueira in the pediatric oncology unit, between February and may 2012. Anthropometric data, body composition and biochemical data collection was conducted 72 hours by following admission. 30 patients were admitted during the study period, the majority were men's $(60 \%)$ with mean age of 7,1 $\pm 4,3$ years. There were $4,2 \%, 3,3 \% 16,7 \%$ of malnutrition according to the indicators weight per age, height per age and body mass index per age, respectively. To adjustment of mid-arm circumference (MAC), Triceps Skinfold Measurement (TSF) and mid-arm muscle circumference (MAMC) was found frequency of malnutrition of $44,8 \%, 57,7 \%$ e $25,9 \%$ respectively. With respect to serum albumin, $48.2 \%$ were classified with malnutrition, the majority of these were patients with hematological malignancies $(65.5 \%)$.With the use of body composition, the largest number of malnourished patients was observed when compared to antoprometric data. Demonstrating the importance of the association of various evaluation methods for better nutritional diagnosis. 


\section{Introdução}

O câncer é uma doença crônico-degenerativa que afeta grande quantidade de indivíduos no mundo todo. O número de novos casos vem aumentando nos últimos anos, sendo, portanto, considerado um problema de saúde pública. Na faixa etária pediátrica, o câncer é definido como toda neoplasia maligna que acomete indivíduos menores de 19 anos (1). Os tumores mais comuns nessa faixa etária são as leucemias, os linfomas e os tumores do sistema nervoso central (2).

A etiologia do câncer infantil é embrionária, apresentando tipo histológico, comportamento biológico, relação com fatores ambientais, evolução clínica e resposta terapêutica diferentes do câncer adulto, o qual tem, na maioria das vezes, um caráter epitelial (3). O tratamento, assim como a própria doença, tem efeitos agressivos para o paciente, deixando o organismo vulnerável e debilitado podendo comprometer o estado nutricional $(4,5)$.

A prevalência de desnutrição ao diagnóstico nas crianças com câncer varia entre $6 \%$ e $50 \%$, dependendo do tipo de tumor, localização, malignidade, estadiamento e critérios adotados para avaliação do estado nutricional (6). Essa prevalência ocorre com maior frequência em crianças com tumores sólidos em estágios avançados, do que em crianças com doença localizada ou leucemias (2).

A desnutrição energética proteica (DEP) na criança com câncer pode ter impacto clínico significativo, estando associada ao aumento da morbidade e da mortalidade, à diminuição da qualidade de vida, da sobrevida e da tolerância ao tratamento. Desse modo, crianças desnutridas ao diagnóstico apresentam resultados significativamente piores do que aquelas que estão eutróficas $(7,8)$.

Por esse motivo, a avaliação e o acompanhamento nutricional em pacientes oncológicos pediátricos são de suma importância e devem ser realizados no momento da internação ou logo após o diagnóstico, sendo repetida com determinada regularidade, para uma maior detecção e controle da desnutrição ou do risco de desnutrição (9).

Tendo em vista as alterações metabólicas provocadas pelo câncer, esse estudo teve o objetivo de avaliar o estado nutricional de pacientes pediátricos em início de tratamento oncológico internados na Unidade de Oncologia Pediátrica do Instituto de Medicina Integral Professor Fernando Figueira (IMIP, Brasil- Recife-PE).

\section{Metodologia}

Estudo transversal do tipo série de casos, realizado com crianças e adolescentes diagnosticados com neoplasia maligna e admitidos para inicio de tratamento na Unidade de Oncologia Pediátrica do Instituto de Medicina Integral Professor Fernando Figueira (IMIP, Brasil, Recife-PE), entre os meses de fevereiro e maio de 2012.

Foram excluídos do estudo crianças e adolescentes impossibilitados de se submeterem a avaliação antropométrica, os portadores de doenças crônicas, síndromes genéticas e pacientes cujo responsável não concordasse com a pesquisa.

Foram registrados dados referentes ao tipo de neoplasia, a partir de informações presentes no prontuário eletrônico do paciente (PEP), e dados coletados através de entrevista com o responsável ou paciente, com questionários previamente estruturados. Os parâmetros antropométricos foram coletados por nutricionista treinada até 72 horas da internação, utilizando as técnicas preconizadas pela Organização Mundial de Saúde (10). O projeto de pesquisa foi aprovado pelo Comitê de Ética e Pesquisa do IMIP, sob o número 2797-11 de acordo com a resolução no 196/96 do Conselho Nacional de Saúde.

Os pacientes foram pesados descalços e com o mínimo de vestimenta, em balança tipo plataforma (Welmy®, W-300A, São Paulo -SP- Brasil) com precisão de 0,100 Kg e 
capacidade de 150,0 Kg. A altura foi aferida em posição ortostática (antropômetro vertical acoplado a balança), com os pacientes de pés descalços e unidos, no centro da plataforma, de costas para o marcador. Foi ainda obtido junto ao responsável dados referentes ao peso de início de tratamento e o peso habitual $(\mathrm{PH})$ para cálculo do percentual de perda ponderal $(\% \mathrm{PP})$ de acordo com a fórmula \%PP $=(\mathrm{PH}-\mathrm{Peso}$ Atual $) / \mathrm{PH} \times 100 \%$.

$\mathrm{O}$ diagnóstico nutricional foi realizado a partir dos indicadores antropométricos, Altura/Idade (A/I), Peso/Idade (P/I) e IMC/Idade (IMC/I), segundo o sexo, tomando-se como base o padrão de referência da Organização Mundial de Saúde e empregando-se o programa WHO AnthroPlus ${ }^{\circledR}$ (11). Os resultados foram expressos em Escores-Z, considerando-se que as crianças abaixo de dois desvios-padrão apresentavam déficits nutricionais e acima de um desvio padrão para o indicador IMC/I, sobrepeso/obesidade, e acima de dois desvio-padrão para P/I, peso elevado para idade (12).

Para avaliação da composição corporal, foi obtida a circunferência do braço (CB) utilizando-se fita métrica inextensível; e para prega cutânea tricipital (PCT) utilizando-se compasso de dobras cutâneas científico Lange ${ }^{\circledR}$ (Beta Technology Incorporated, Cambridge - MD) com pressão constante de $10 \mathrm{gr} / \mathrm{mm} 2$. As medidas foram realizadas no ponto médio entre o olecrano e o acrômio no braço não dominante. Para a realização desta medida o participante permaneceu em pé, com os braços relaxados ao lado do corpo de acordo com técnica proposta por Frisancho (1990) (13). Através das medidas de CB e PCT, foi calculada a circunferência muscular do braço $(\mathrm{CMB})$ através da fórmula: $\mathrm{CMB}=\mathrm{CB}-($ PCTx0,314).

As medidas de CB, PCT e CMB foram avaliadas utilizando-se a adequação percentual em relação ao percentil 50 da referência de Frisancho (13), definindo-se com depleção o valor de adequação percentual $<90 \%$ e, como eutrofia o valor de adequação percentual $>90 \%$.

A coleta de sangue destinada à análise da albumina precedeu o início de qualquer tratamento radio ou quimioterápico, sendo colhido pela manhã após 12 horas de jejum. Alíquota de $5 \mathrm{ml}$ de soro foi obtida por centrifugação e encaminhada à laboratório conveniado ao IMIP. A albumina foi analisada pelo método colorimétrico com verde bromocresol, medida no aparelho Architec ${ }^{\circledR}$, por automação. Para análise adotou-se como ponto de corte menor que $3,2 \mathrm{~g} / \mathrm{dL}$ como indicativo de desnutrição para pacientes críticos oncológicos pediátricos Para análise adotou-se como ponto de corte menor que $3,2 \mathrm{~g} / \mathrm{dL}$ como indicativo de desnutrição para pacientes críticos oncológicos pediátricos (14).

Os dados foram digitados no programa Excel para Windows ${ }^{\circledR}$. As análises foram realizadas no Programa SPSS versão 13.0. As variáveis contínuas foram testadas quanto à normalidade pelo teste de Kolmogorov Smirnof. Para as variáveis com distribuição normal, utilizou-se o teste t de Student para comparar dois grupos independentes e a Análise de Variância (ANOVA) de uma via para mais de dois grupos. Para as variáveis com distribuição não normal, utilizou-se teste de Mann Whitney para comparar dois grupos. Adotou-se nível de significância de 5\%.

\section{Resultados}

Foram admitidos no período do estudo 30 pacientes. A maioria era do sexo masculino (60\%) com média de idade de 7,1 $\pm 4,3$ anos. As neoplasias mais frequentes foram: leucemia $(29,9 \%)$, seguida por tumores de sistema nervoso central $(26,7 \%)$ e linfomas $(13,3 \%)$, os demais tumores foram sólidos que totalizaram 29,7\% da amostra (Figura 1). 


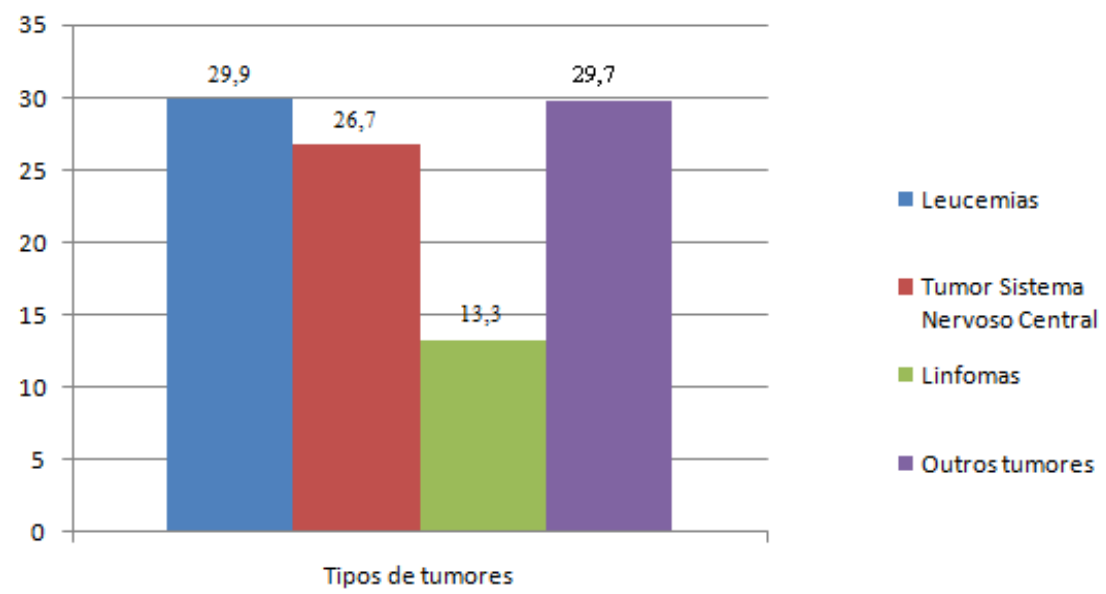

Figura 1- Frequências de neoplasias em crianças e adolescentes admitidos em Hospital de referência. Recife -PE, Brasil (2012).

O estado nutricional foi avaliado utilizando os parâmetros de peso, altura e idade. Os resultados apontam 4,2\%, 3,3\% e 16,7\% de déficit nutricional de acordo com os indicadores de P/I, A/I e IMC/I respectivamente. Enquanto que o excesso de peso esteve presente em $13,3 \%$ dos pacientes avaliados segundo o IMC/I.

Os dados referentes à composição corporal evidenciam maiores frequências de desnutrição quando comparados à avaliação segundo os escores $\mathrm{Z}$ dos indicadores antropométricos. Encontrando-se, frequências de desnutrição de 44,8\%;57,7\% e 25,9\% para os parâmetros de CB, PCT e CMB respectivamente, evidenciando maior depleção de tecido adiposo do que muscular. (57,7\% versus $25,9 \%$ avaliados pela PCT e CMB, respectivamente) (Figura 2). Com relação à albumina sérica, $48,2 \%$ foram classificados com desnutrição, destes a maioria eram pacientes com tumores hematológicos $(65,5 \%)$.

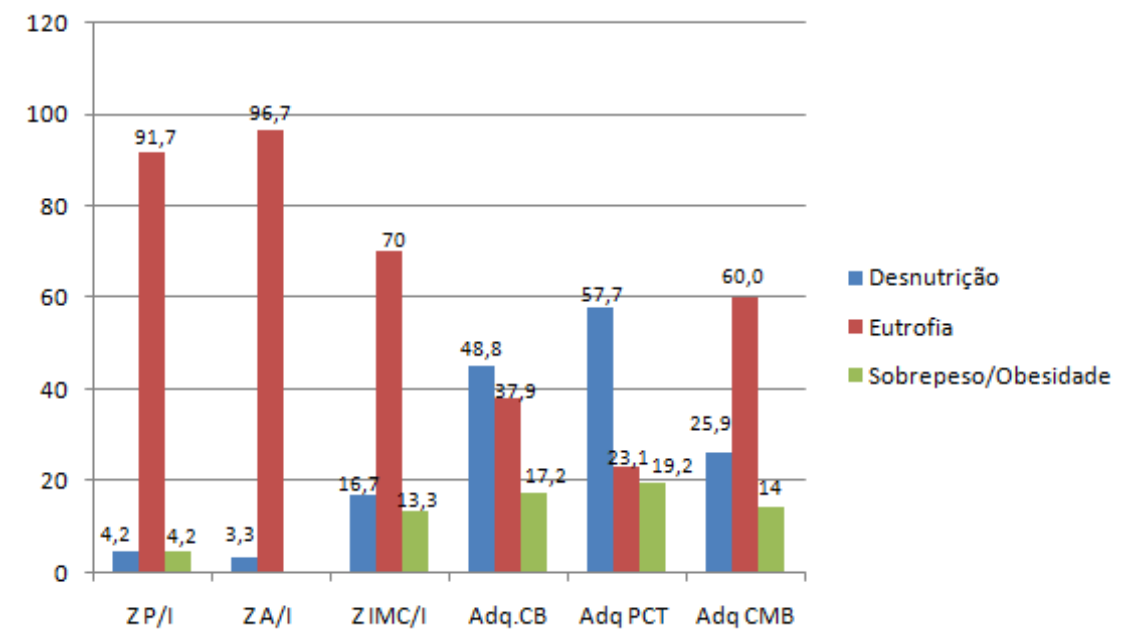

Figura 2 - Estado nutricional de crianças e adolescentes com câncer em inicio de tratamento, segundo os índices antropométricos e a avaliação de composição corporal. Recife - PE Brasil (2012). * P/I: Peso/ Idade; A/I: Altura/ idade; IMC/I: Índice de massa corporal CB: Circunferência do braço; PCT: Prega cutânea tricipital; CMB: Circunferência muscular do braço.

A tabela 1 apresenta as médias das variáveis antropométricas de acordo com a presença do risco nutricional (Escore $\mathrm{Z}$ de $\mathrm{IMC} / \mathrm{I}<-1 \mathrm{dp}$ ). Os pacientes com risco nutricional apresentaram menores medias de Escore Z P/I, percentual de adequação de peso ideal, CB, e $\mathrm{CMB}$, com diferenças estatisticamente significantes $(\mathrm{p}<0,05)$. Segundo a Tabela 2 , os 
pacientes com diagnóstico de tumor sólido possuíram maiores médias em relação às crianças com tumor hematológico, porém a única diferença estatisticamente significante foi em relação à albumina sérica $(\mathrm{p}<0,05)$.

Tabela 1. Variáveis antropométricas de acordo com a presença do risco nutricional das crianças e adolescentes com câncer admitidos em Hospital de referência no Recife -PE, Brasil (2012)

\begin{tabular}{lccc}
\hline \multicolumn{1}{c}{ Variável } & \multicolumn{2}{c}{ Risco nutricional* } & \\
& $\begin{array}{c}\text { Sim } \\
\text { Média } \pm \text { DP } \\
\mathbf{N = 1 2}\end{array}$ & $\begin{array}{c}\text { Não } \\
\text { Média } \pm \text { DP } \\
\mathbf{N = 1 8}\end{array}$ & Valor de p \\
& $-1,14 \pm 0,69$ & $0,259 \pm 1,16$ & \\
\hline Escore Z de P/I & $0,210 \pm 1,11$ & $0,105 \pm 1,29$ & $\mathbf{0 , 0 2 0}$ \\
Escore Z de A/I & $83,80 \pm 4,63$ & $104,34 \pm 12,36$ & 0,821 \\
Adequação Peso ideal $(\%)$ & $86,07 \pm 9,35$ & $102,11 \pm 15,62$ & $\mathbf{0 , 0 0 1}$ \\
Adequação de CB(\%) & $65,60 \pm 23,29$ & $90,47 \pm 37,14$ & $\mathbf{0 , 0 0 4}$ \\
Adequação de PCT $(\%)$ & $91,2 \pm 8,87$ & $101,21 \pm 10,88$ & 0,071 \\
Adequação de CMB $(\%)$ & $9,70 \pm 6,29$ & $11,52 \pm 9,53$ & $\mathbf{0 , 0 1 8}$ \\
Perda de peso \% & $3,12 \pm 0,56$ & $3,18 \pm 0,54$ & 0,630 \\
Albumina (mg/dL) & Ald & 0,773 \\
\hline
\end{tabular}

*Risco nutricional valor de Escore Z de IMC/I<-1; DP: desvio-padrão.

Tabela 2. Médias com relação a crianças e adolescentes com tumor sólido e tumor hematológico admitidos em Hospital de referência no Recife -PE, Brasil (2012)

\begin{tabular}{lccc}
\hline \multicolumn{1}{c}{ Variável } & $\begin{array}{c}\text { Tumor } \\
\text { sólido } \\
\text { Média } \pm \text { DP } \\
\mathbf{n 1 3}\end{array}$ & $\begin{array}{c}\text { Tumor } \\
\text { hematológico } \\
\text { Média } \pm \mathbf{D P} \\
\mathbf{n 1 7}\end{array}$ & Valor de $\mathbf{p}$ \\
\hline Escore Z de P/I & $-0,29 \pm 1,09$ & $-0,50 \pm 1,37$ & 0,684 \\
Escore Z de A/I & $0,11 \pm 1,26$ & $0,18 \pm 1,16$ & 0,871 \\
Peso ideal (\%) & $97,83 \pm 15,72$ & $93,89 \pm 12,26$ & 0,462 \\
Adequação de CB(\%) & $97,95 \pm 16,39$ & $91,96 \pm 13,89$ & 0,312 \\
Adequação de PCT (\%) & $81,00 \pm 32,42$ & $80,80 \pm 37,80$ & 0,989 \\
Adequação de CMB (\%) & $98,15 \pm 9,72$ & $95,82 \pm 13,01$ & 0,599 \\
Perda de peso (\%) & $11,73 \pm 9,71$ & $8,79 \pm 3,43$ & 0,340 \\
Albumina (mg/dL) & $3,36 \pm 0,52$ & $2,91 \pm 0,46$ & $\mathbf{0 , 0 2 3}$ \\
Escore Z de IMC/I & $-0,4994 \pm 1,67$ & $-0,84 \pm 1,41$ & 0,553 \\
\hline
\end{tabular}

\section{Discussão}

De acordo com os resultados observou-se baixa frequência de déficit ponderal e estatural na amostra avaliada, segundo indicadores A/I, P/I e IMC/I. Entretanto, as medidas de composição corporal revelaram maiores déficits nutricionais, com maior comprometimento da reserva adiposa ao diagnóstico.

Resultados inferiores aos nossos foram encontrados por Carraro et al. (15) estudando 19 crianças e adolescentes internados no Hospital de Clínicas de Porto Alegre, segundo os valores de escore-z de IMC/I obtiveram 10,5\% dos pacientes com magreza. Vale ressaltar que esses pacientes não foram avaliados logo na admissão e sim após pelo menos um ciclo de tratamento antineoplásico, o que poderia ter influenciado seu estado nutricional.

Crianças portadoras de neoplasias malignas em geral são desnutridas ao serem diagnosticadas e a avaliação nutricional utilizando medidas antropométricas de idade, peso e altura quando utilizadas de forma isolada podem não ser adequadas, sendo necessária a associação com outros parâmetros. A utilização da composição corporal pode ser uma 
alternativa, visto que as neoplasias malignas podem influenciar uma série de alterações corporais decorrentes do tumor ou mesmo do tratamento. Neste contexto, a CMB e a PCT podem ser úteis para complementar o diagnóstico nutricional, sendo utilizada para estimativa da reserva de massa magra e de gordura, respectivamente $(2,16)$

Em recente casuística realizada no Reino Unido, a prevalência de desnutrição também foi maior quando utilizada composição corporal. Foram avaliados 74 pacientes pediátricos recém-diagnosticados com câncer e de acordo com IMC/I a prevalência de desnutrição foi de $9,5 \%$ e de acordo com a PCT foi $13,5 \%$ (17)

Garófalo et al. (16), ao estudar crianças com tumores sólidos e hematológicos no primeiro mês de tratamento (fase de indução da remissão da doença) internados no Instituto de Oncologia Pediátrica da Universidade Federal de São Paulo, encontraram prevalências de déficit pelo indicador IMC/I em 18,9\%, bem como pela CMB e pela PCT de 35,4\% e 40,2\%, respectivamente. Ao utilizar as relações IMC/I ou P/E pode-se subestimar a prevalência de desnutrição em comparação com a PCT e a CMB $(9,16)$.

Medidas de peso subestimaram as prevalências de desnutrição quando compradas a medidas de composição corporal. Tais resultados corroboram com dados encontrados na literatura que evidenciam que medidas de prega e circunferências, tais como: PCT, $\mathrm{CB}$ e $\mathrm{CMB}$, são mais sensíveis para detectar o comprometimento nutricional em crianças e adolescentes com câncer, quando comparadas aos indicadores que utilizam peso e estatura (2,15, 17-19).

Quando utilizados os parâmetros de composição corporal, os resultados corroboram com estudo conduzido por Araújo et al.(19), no mesmo centro de referência, com crianças e adolescentes em início de tratamento antineoplásico. Os autores encontraram baixa frequência de déficit ponderal e estatural, segundo indicadores A/I, P/I e IMC/I quando comparado às medidas de composição corporal. Pelo IMC /I encontrou-se 13,3\% de déficit nutricional, porém pela CMB e pela PCT, tais parâmetros apontaram para depleção em 32\% e $68 \%$, respectivamente.

Lemos et al. (20) em estudo observacional transversal avaliaram 1.154 crianças e adolescentes com tumores hematológicos e sólidos internados nas enfermarias ou em unidades de terapia intensiva do Instituto de Oncologia Pediátrica do Departamento de Pediatria da Universidade Federal de São Paulo e encontraram que 10,8\% apresentavam baixo peso pelo IMC/I, enquanto que ao utilizar métodos de composição corporal o déficit aumentou para 27,2\% utilizando a PCT e 24,7\% utilizando a CMB (20). Em uma casuística realizada na cidade de Casablanca em Marrocos com 100 crianças e adolescentes em início de tratamento oncológico, foram diagnosticados $33 \%$ dos pacientes com desnutrição pelo IMC/I, porém, ao utilizar as medidas de PCT e CMB, obteve-se déficit em $50 \%$ e $39 \%$ da amostra, respectivamente. Evidencia-se assim, que quando se utilizam as medidas de composição corporal são obtidas maiores frequências de déficit nutricional (21).

No presente estudo, os pacientes com diagnóstico de tumor sólido possuíram maiores médias dos indicadores antropométricos quando comparados às crianças com tumor hematológico, porém apenas os níveis séricos de albumina apresentaram diferença estatisticamente significante. Estes dados não corroboram com outros estudos que apontam maior depleção de massa muscular e adiposa entre crianças portadoras de tumores sólidos $(2,16,20,21)$. Baseados nisto alguns autores reforçam a necessidade da utilização de medidas de composição corporal devido às dificuldades na obtenção do peso real destes pacientes, usualmente mascarados por edema e o tamanho do tumor $(20,21)$.

A frequência de déficit nutricional avaliado pelos níveis séricos de albumina foram superiores aos encontrados pelos parâmetros antropométricos e de composição corporal e dentre estes, os portadores de tumores hematológicos foram os mais afetados. Entretanto, Tazi et al. (21) avaliando 100 crianças e adolescentes com tumores sólidos e 
hematológicos, obtiveram que apenas $28 \%$ foram diagnosticados com desnutrição por esse parâmetro e observaram níveis mais baixos de albumina em pacientes com neoplasia hematológica.

A albumina é uma proteína visceral recomendada na avaliação do estado nutricional de pacientes hospitalizados. No entanto, em pacientes portadores de neoplasia maligna, este parâmetro isolado é pouco sensível e inespecífico como marcador do estado nutricional, pois condições clínicas que são muito frequentes nesses pacientes, como estado de hidratação, presença de inflamação e infecção e medicamentos, podem levar a mudanças na concentração sérica dessa proteína (22).

\section{Considerações finais}

No presente estudo foram verificados, já na admissão, déficits nutricionais importantes, porém os resultados apresentados são limitados, considerando o reduzido número da amostra. No entanto, ainda são limitados na literatura estudos sobre avaliação do estado nutricional de pacientes oncológicos pediátricos utilizando parâmetros de composição corporal para uma avaliação mais precisa destes pacientes. Contudo, estes pacientes têm uma maior tendência à desnutrição em decorrência da própria patologia e dos efeitos colaterais do tratamento, o que consequentemente pode levar a um aumento da morbidade e da mortalidade, a uma diminuição da qualidade de vida, da sobrevida e da tolerância ao tratamento. Desta forma, é importante a realização de estudos futuros visando uma melhor avaliação do estado nutricional para que medidas adequadas de intervenção nutricional sejam adotadas.

Conflito de interesses: A autora declara não haver conflito de interesses

\section{Referências}

1. Sousa CNS, Santiago CMC, Pereira WO, Morais FRR. Epidemiological profile of cancer: study in oncology and hematology hospital. Rev Enferm UFPE online. 2012;6(5):968-976.

2.Garófalo A. Diretrizes para Terapia Nutricional em Crianças com Câncer em Situação Crítica. Rev. Nutr.2005; 18: 513-527.

3. Bianchi A, Camargo B. O papel do pediatra frente à criança com câncer. In: Camargo B, Lopes LF. Pediatria Oncológica. Noções Fundamentais para o Pediatra; $1^{\text {a }}$ Edição, São Paulo: Editora Lemar; 2000. p. 1-6.

4. Viana MB, Murao M, Ramos G, Oliveira HM, Carvalho RI, Bastos M, et al. Malnutrition as a prognostic factor in lymphoblastic leukaemia: a multivariate analysis. Arch. Dis. Child. 1994;71: 304-310.

5. Watters AL, Epstein JB, Agulnik M, Oral complications of targeted cancer therapies: A narrative literature review. Oral Oncology. 2011; 47: 441-448.

6. Ladas EJ, Sacks N, Meacham L, Henry D, Enriquez L, Lowry G, et al. A Multidisciplinary Review of Nutrition Considerations in the Pediatric Oncology Population: A Perspective From Children's Oncology Group. Nutr. Clin. Pract. 2005; 20(4): 377-393.

7. Garófalo A, Petrilli AS. Terapia nutricional em oncologia. In: Fundamentos da terapia nutricional em pediatria. São Paulo: Savier. 2002 p. 214-234.

8. Paul CJ. Nutritional Status As a Prognostic Indicator for Pediatric Malignancies. Journal of Clinical Oncology, 2014;32(13):1293-1294.

9. Caprara GL, Ricalde SR, Santos JS. Características nutricionais dos pacientes oncológicos pediátricos do Hospital Geral de Caxias do Sul. Pediatria(São Paulo) 2009;31(4):234-241. 
10.WHO, Physical status: the use and interpreteition of anthhorpometry . Report of a WHO Expert Committee. Geneva; 1995( Technical Report Series, 854)

11.World Health Organization. Multicentre Growth Reference Study Group: Who Child Growth Standards: Length/height-for-age, weight-for-age, weight-for-length, weight-for-height and body mass index-for-age: Methods and development. Geneva, World Health Organization, 2006.

12. World Health Organization. Anthro for personal computers, version 3.1, 2010: Software for assessing growth and development of the world's children. Geneva: WHO, 2010.

13. Frisancho AR. Anthropometic standards for the assessment of growth and nutrional status. Ann Arbor, Mich: University of Michigen Press, 1990.

14. Instituto Nacional Do Câncer. Coordenação de Prevenção e Vigilância de Câncer. Câncer da criança e adolescente no Brasil: dados dos registros de base populacional e de mortalidade. Instituto Nacional de Câncer. Rio de Janeiro: INCA, 2009 disponível em: http://www.inca.gov.br. Acessado em 10 de agosto de 2014.

15. Carraro JL, Schwartz R, Behling EB. Comparação do estado nutricional obtido através de antropometria e bioimpedância em crianças e adolescentes submetidos a tratamento quimioterápico em um hospital no Sul do Brasil. Revista HCPA. 2012; 32(1): 35-41.

16.Garófolo A, Lopez FA, Petrilli AS. High prevalence of malnutrition among patients with solid nonhematological tumors as found by using skinfold and circunference measurements. São Paulo Med J 2005; 123(6):277-281

17. Iniesta RR, McKenzie J, Ilenia P, Brougham M, David W. Nutritional status of paediatric cancer patients from the UK: a prospective cohort study .The FASEB Journal. 2014; 28(1): 825-827.

18. Motil KJ. Sensitive Measures of Nutritional Status in Children in Hospital and in the Field. Int J Câncer 1998; 78:2-9.

19. Araújo LL, Barbosa JM, Ribeiro APG, Santos ACO, Pedrosa F. Nutritional status, dietary intake and serum levels of vitamin C upon diagnosis of cancer in children and adolescents. Nutr Hosp. 2012; 27(2): 496-503.

20. Lemos PSM, Oliveira FLC, Caran LMN. Nutritional status of children and adolescents atdiagnosis of hematological and solid malignancies. Rev Bras Hematol. Hemoter. 2014;36(6): 420-423.

21. Tazi I, Hidane Z, Zafad S, Harif M, Benchekroun S, Ribeiro R. Nutritional Status at Diagnosis of Children with Malignancies in Casablanca. Pediatr Blood Cancer 2008; 51:495-498.

22. Barbosa JM, Ribeiro APG. Cancer. In: Vasconcelos MJOB, Barbosa JM, Pinto ICS, Lima TM, Araujo AFC. Nutrição Clínica Obstetrícia e Pediatria; $1^{a}$ edição, Rio de Janeiro: Editora Medbook 2011, p 445470. 